\title{
RECURRENCE OF AFEBRILE STATUS EPILEPTICUS
}

Risk factors for recurrence of afebrile status epilepticus (SE), in residents of Rochester, MN, were determined by researchers at Columbia University, New York; Harvard School of Public Health, Boston, MA; and Mayo Clinic, Rochester, MN. Among 183 episodes of first afebrile SE recorded between Jan 1, 1965, and Dec 31, 1984, the risk of recurrence was $31.7 \%$ over a 10 -year follow-up period. SE was acute symptomatic in 101 $(55.2 \%)$ cases and unprovoked in $82(44.8 \%)$. Most recurrences $(81.8 \%)$ occurred during the first 2 years after the first episode. Among children, the risk for recurrence was $13.1 \%$ at 1 year and $16.9 \%$ at 2 years. The risk of recurrence in the total population was $100 \%$ for progressive symptomatic SE, $23.6 \%$ for remote symptomatic SE, $26.1 \%$ for idiopathic/cryptogenic SE, and $26.3 \%$ for acute symptomatic SE. Risk of recurrence was not related to patient age and duration of SE. It was significantly greater for females vs males $(39.3 \%$ vs $24.6 \%, \mathrm{P}=0.02)$. Risk was reduced in patients who responded to the first AED used to treat $\mathrm{SE} ; 14.7 \%$ recurrence in those with response vs $38.2 \%$ in those with poor response, $\mathrm{P}=0.01$. Predictors of recurrence of $\mathrm{SE}$ were sex, etiology, seizure type, and response to the first anticonvulsant drug. Risk was increased 2.3-fold for females, and 2.4fold for progressive symptomatic SE cf idiopathic /cryptogenic SE. It was decreased for partial seizures without generalization, and for SE that responded to the first treatment. (Hesdorffer DC, Logroscino G, Cascino GD, Hauser WA. Recurrence of afebrile status epilepticus in a population-based study in Rochester, Minnesota. Neurology July 2007;69:73-78). (Reprints: Dr DC Hesdorffer, GH Sergievsky Center, $630 \mathrm{~W}, 168^{\text {th }}$ St, P\&S Box 16, New York, NY 10032).

COMMENT. Status epilepticus (SE) was defined as a single clinical seizure lasting more than $30 \mathrm{~min}$ or repeated seizures over a period of more than $30 \mathrm{~min}$ without recovery of consciousness. Some authorities include seizures of shorter duration, $10 \mathrm{~min}$ or longer. Except for cases of progressive degenerative cerebral disease that were associated with a $100 \%$ risk of SE recurrence, patients with SE have a 1 in 4 chance of recurrence, regardless of age or duration of the initial SE episode. In a population-based study, one-third has a recurrence of SE, and the risk is doubled for females and for progressive symptomatic SE.

Efficacy of IV midazolam for status epilepticus was determined in a nationwide retrospective multicenter study reported from the Department of Pediatrics, Tokyo Women's Medical University, Japan. (Hayashi K et al. Pediatr Neurol June 2007;36:366-372). Among 358 inpatients treated, mean age 48.6 months, 195 had epilepsy and 163 had an acute symptomatic disease (encephalitis or encephalopathy in 88). A bolus dose of midazolam $(0.25+/-0.21 \mathrm{mg} / \mathrm{kg})$ followed when necessary by continuous infusion $(0.26+/-0.25$ $\mathrm{mg} / \mathrm{kg} / \mathrm{hr}$ ) was effective in $56.6 \%$ cases, and final seizure suppression was obtained in $64.5 \%$. Midazolam was less effective in patients treated late and more than 3 hours after seizure onset, especially those with epilepsy. Adverse events occurred in $19.2 \%$ of those receiving the bolus injection only, and in $28.2 \%-58.6 \%$ of those who required continuous infusion in doses below or above $0.4 \mathrm{mg} / \mathrm{kg} / \mathrm{hr}$, respectively. Respiratory suppression occurred in 86 cases; symptoms were judged related to the underlying disease or the SE in 57. Ten deaths were also connected to the disease and not the treatment. Midazolam is not approved and has only off-label use. 
In the United States, lorazepam, with its longer half-life compared to diazepam, is considered most appropriate as initial treatment for generalized tonic-clonic status epilepticus. (Wheless JW et al. Eds. Treatment of pediatric epilepsy: Expert opinion 2005. J Child Neurol Dec 2005;20(Suppl 1):S46-S49). If the seizure continues despite the maximum dose of benzodiazepine, this is followed with IV fosphenytoin as the most appropriate second drug selection.

\section{TOPIRAMATE AND VALPROATE COMPARED IN TREATMENT OF JUVENILE MYOCLONIC EPILEPSY}

A pilot, randomized, controlled trial comparing topiramate $(n=19)$ and valproate $(\mathrm{n}=9)$ in adolescents/adults with juvenile myoclonic epilepsy (JME) was conducted in the Childrens Hospitals of Denver, $\mathrm{CO}$, and Cincinnati, $\mathrm{OH}$. Of patients completing a 26 week trial of these agents titrated to optimal effect, 8 of $12(67 \%)$ in the topiramate group and 4 of $7(57 \%)$ in the valproate group were seizure-free during the 12 -week maintenance period. Median daily doses were $250 \mathrm{mg}$ topiramate and $750 \mathrm{mg}$ valproate. Adverse events resulted in discontinuance of treatment in $2(11 \%)$ of topiramate-treated and one $(11 \%)$ valproatetreated patients. Valproate had a higher incidence of systemic toxicity than topiramate, including nausea $(33 \%)$, weight gain $(22 \%)$, increased appetite $(22 \%)$, alopecia $(33 \%)$ and rash $(22 \%)$. A double-blind trial of topiramate in JME is recommended. (Levisohn PM, Holland KD. Topiramate or valproate in patients with juvenile myoclonic epilepsy: A randomized open-label comparison. Epilepsy \& Behavior 2007;10:547-552).

COMMENT. Topiramate may be an effective alternative treatment to valproate for juvenile myoclonic epilepsy (JME). An expert opinion panel rated valproate and lamotrigine as most appropriate treatment for JME in males, and lamotrigine followed by topiramate in females. (Wheless JW et al. J Child Neurol 2005;20(Suppl 1):S42-S43). Zonisamide and levetiracetam are rated sometimes appropriate. Valproate is not recommended in adolescent and young adult females with JME because of risk of teratogenicity and potential harmful effects on the infant's neuropsychological development (Eriksson $\mathrm{K}$ et al. Epilepsy Res $2005 ; 65: 189-200)$.

Levetiracetam in severe myoclonic epilepsy of infancy (Dravet syndrome). Levetiracetam add-on therapy was administered in doses of 10 up to $50-60 \mathrm{mg} / \mathrm{kg} /$ day in 28 children (mean age $9.4+/-5.6$ years) with SMEI (16 with $S C N 1 A$ mutations). Mean dose was 2,016 mg/day. Tonic-clonic, myoclonic, and focal seizures responded in 60-64\% cases, and absence seizures in $44 \%$. Myoclonic seizures were reduced from 21 to 3 per week ( $\mathrm{p}=.002$ ), and tonic-clonic from 3 to 1 week $(\mathrm{p}=.0001)$. Dravet syndrome is a drug-resistant epilepsy with onset in the first year of life. It is characterized by prolonged and repeated febrile and afebrile generalized or focal convulsive seizures. Myoclonic and partial seizures develop between 1 and 4 years of age. Psychomotor development, initially normal, is delayed in the $2^{\text {nd }}$ year. Seizures are usually refractory to treatment. 